\title{
A hybrid control against species invasion in the chemostat
}

\author{
Fatima-Zahra Tani, Alain Rapaport and Térence Bayen
}

\begin{abstract}
In this work, we consider the classical chemostat model with the objective to limit the invasion of a new species having negative effect on the resident one, playing with the removal rate. We study the resilience of the system to the apparition of an invader, and propose a new concept of weak resilience when the system cannot return and stay at its original state (whatever is the removal rate). The weak resilience guarantees that the measure of the time for which the density of the resident species is above a given threshold is infinite. We show that this can be achieved by a hybrid controller with very few knowledge on the dynamics of the system. As it is not possible to eradicate totally the invasive species, the controller makes the resident species return indefinitely many times above the desired threshold, and the solutions converge asymptotically to periodic solutions. We illustrate the effectiveness of the proposed controller on numerical simulations.
\end{abstract}

\section{INTRODUCTION}

The well known chemostat model [20], [15] describes the microbial growth in continuous culture [18] and is also often used to represent continuously stirred tank reactors [5]:

$$
\begin{aligned}
\dot{s} & =-\frac{1}{Y} \mu(s) x+D\left(s_{i n}-s\right), \\
\dot{x} & =(\mu(s)-D) x .
\end{aligned}
$$

The variables $s$ and $x$ denote the concentrations of substrate and biomass, respectively. The operating parameters are the input concentration of substrate $s_{i n}$ and the removal rate $D$ (we assume that the effective removal rate is the same on both concentrations, which amounts to neglect mortality or attachment of bacteria). The parameter $Y$ and the function $\mu(\cdot)$ are the yield conversion and the specific growth rate, that we assume here to be increasing with $\mu(0)=0$ (as it is often the case in microbiology [18]). Without loss of generality, one can take $Y=1$ (at the price to change the biomass unit). In many industrial applications, bacteria are expected to provide ecosystem services (such as water purification) or produce substances of economic interest (in pharmacology for instance). It is often of primer importance to maintain the concentration of the biomass $x$ at or above a given threshold. One can easily check that a positive equilibrium $\left(s^{\star}, x^{\star}\right)$ of (1) verifies $\mu\left(s^{\star}\right)=D$ and $x^{\star}=Y\left(s_{\text {in }}-s^{\star}\right)$. Typically, bioreactors are operated at steady state choosing the removal rate $D$ to be equal to a nominal value $D^{\star}:=\mu\left(s_{\text {in }}-x^{\star} / Y\right)$ where $x^{\star}$ is the desired biomass concentration (see for instance [5], [15]). However, it is also well known that open systems are subject to bacterial contamination or genetic evolution. Therefore, another bacterial species can appear in

F.-Z. Tani and A. Rapaport are with MISTEA, Univ. Montpellier, INRA, Montpellier SupAgro, France alain.rapaporteinra.fr, fatima-zahra.taniesupagro.fr

T. Bayen is with IMAG, Univ. Montpellier, CNRS, France terence.bayen@umontpellier.fr the chemostat system at any time. The chemostat model with more than one species (here two, assuming without loss of generality that the yield coefficients $Y_{i}$ are equal to 1):

$$
\left\{\begin{array}{l}
\dot{s}=-\mu_{1}(s) x_{1}-\mu_{2}(s) x_{2}+D\left(s_{i n}-s\right), \\
\dot{x_{1}}=\left(\mu_{1}(s)-D\right) x_{1}, \\
\dot{x_{2}}=\left(\mu_{2}(s)-D\right) x_{2},
\end{array}\right.
$$

has been widely studied in the literature [2], [20], [14], [13], [15]. We also assume that the removal rates of bacteria are identical). The main result is the so-called "Competitive Exclusion Principle" [16], [3], [12], which states that (generically) at most one species survives at steady state. Then, depending on the characteristics of the invading species, this one can settle and eradicate the resident species, which can be catastrophic for operators if the new species does not provide similar ecosystem services or by-products. However, competing species often present compromises. Typically, a species could be specialized for low substrate concentrations while another one could be for larger concentrations. In such cases, it has been shown that applying a periodic timevarying removal rate $D(\cdot)$ could allow coexistence of both species [19], [11], [22]. It appears that the design of such time-varying controls requires the perfect knowledge of the growth functions $\mu_{i}(\cdot)$. In addition, to our best knowledge, the performances in terms of services that continue to be provided by the resident species, have not been studied in the literature. This is precisely the objective of the present work. In particular, we shall see that hybrid controllers can be defined without a precise knowledge of the functions $\mu_{i}(\cdot)$ allowing coexistence of both species. In addition, for the corresponding solution, the time spent by the density of the resident species above a given threshold is infinite.

The paper is organized as follows. In Section II, we setup assumptions and study the resilience of the chemostat model (1) in presence of an invading species, considering system (2). We introduce here a new concept of weak resilience. In Section III, we present recent results about the design of time-varying removal rates that make the system weak resilient. We then show that such open-loop controls can indeed be synthesized by a hybrid feedback controller. Section IV]illustrates with numerical simulations the behavior of the proposed controller and discusses the role of its parameters.

\section{RESILIENCE ANALYSIS}

For system (2), let us consider the following (classical) assumptions:

Assumption A1: The functions $\mu_{i}(\cdot)(i=1,2)$ are $C^{1}$ increasing functions with $\mu_{i}(0)=0$. 
Let us also introduce the break-even concentrations:

$$
\lambda_{i}(D):=\sup \left\{s \in\left[0, s_{i n}\right] ; \mu_{i}(s)<D\right\}, \quad i=1,2 .
$$

Then, the Competitive Exclusion Principle (CEP) [12], [20], [15] states as follows.

Theorem 1 (CEP): Under Assumption A1, if $\lambda_{i}(D)<$ $\min \left(\lambda_{j}(D), s_{i n}\right)$ for $i \neq j, i, j \in\{1,2\}$, then any positive solution of (2) converges asymptotically to the equilibrium $\left(s^{\star}, x_{1}^{\star}, x_{2}^{\star}\right)$ with $s^{\star}=\lambda_{i}(D), x_{i}^{\star}=s_{i n}-\lambda_{2}(D)$ and $x_{j}^{\star}=0$.

Therefore, several cases appear:

- if $\mu_{2}(s)>\mu_{1}(s)$ for any $s>0$, then one has $\lambda_{2}(D)<\lambda_{1}(D)$ for any $D \in\left(0, \mu_{1}\left(s_{i n}\right)\right)$, and the resident species never survives under invasion by species 2 .

- On the opposite, if $\mu_{2}(s)<\mu_{1}(s)$ for any $s>0$, the resident species is safe in presence of such an invader (with $D<\mu_{1}\left(s_{i n}\right)$ to avoid the washout equilibrium).

- However, as mentioned in the introduction, it often happens that species are complementary: if the invader can settle and eradicate the resident species for small values of $s$, the issue of the competition is reversed under large concentrations of $s$, what we shall consider in the sequel with the following assumption:

Assumption A2: There exists $\bar{s} \in\left(0, s_{i n}\right)$ such that

$$
\left(\mu_{1}(s)-\mu_{2}(s)\right)(s-\bar{s})>0, \quad s \in\left[0, s_{i n}\right] \text { and } s \neq \bar{s} .
$$

Denote then $\bar{D}:=\mu_{1}(\bar{s})=\mu_{2}(\bar{s})$.

For any $D \in\left(\bar{D}, \mu_{1}\left(s_{i n}\right)\right)$, the resident species 1 wins the competition and its density at steady state is equal to $x_{1}^{*}:=$ $s_{i n}-\lambda_{1}(D)$. Therefore the number

$$
\bar{x}_{1}:=s_{\text {in }}-\lambda_{1}(\bar{D})=s_{\text {in }}-\bar{s}>0 .
$$

is the largest density level that the system can maintain in presence of the invader 2 under constant removal rate. However, one may wonder if it is possible to maintain higher levels with time varying removal rate $D(\cdot)$ taking values in an interval $\left[D_{m}, D_{M}\right]$ with

$$
0<D_{m}<\bar{D}<\mu_{1}\left(s_{i n}\right)<D_{M} .
$$

One can easily check that the domain

$$
\left\{\left(s, x_{1}, x_{2}\right) \in \mathbb{R}_{+}^{3} ; s+x_{1}+x_{2}=s_{i n}\right\},
$$

is invariant by (2) and attractive for any persistently exciting control $D(\cdot)$. By persistently exciting control, we mean any non-negative function $D(\cdot)$ such that $\int_{0}^{+\infty} D(t) d t=+\infty$ (see [5]). Considering that (1) is already at steady state before invasion, and that the quantity $x_{2}$ at invasion is very small, we shall consider in the sequel the reduced dynamics on this domain, that is, the planar dynamics

$$
\dot{x}=f(x, D):=\left[\begin{array}{l}
\left(\mu_{1}\left(s_{i n}-x_{1}-x_{2}\right)-D\right) x_{1} \\
\left(\mu_{2}\left(s_{i n}-x_{1}-x_{2}\right)-D\right) x_{2}
\end{array}\right],
$$

defined on the invariant set

$$
\mathscr{S}:=\left\{x \in \mathbb{R}_{+}^{2} ; x_{1}+x_{2} \leq s_{\text {in }}\right\} .
$$

Consider then a threshold $x_{1}^{r} \in\left(\bar{x}_{1}, s_{i n}\right)$ satisfying

$$
0<D_{m}<\mu_{1}\left(s_{i n}-x_{1}^{r}\right),
$$

and define a subset $\mathscr{K}\left(x_{1}^{r}\right)$ of $\mathscr{S}$ as:

$$
\mathscr{K}\left(x_{1}^{r}\right):=\left\{x \in \mathscr{S} ; x_{1} \geq x_{1}^{r} \text { and } x_{2}>0\right\} .
$$

Let us now formulate the invasion problem in terms of resilience. Assume that, at the initial time, species 1 is alone with a density level equal to $x_{1}^{r}$ ensured by the choice of the constant dilution rate $D^{r}:=\mu_{1}\left(s_{i n}-x_{1}^{r}\right)<\bar{D}$, and that species 2 (satisfying Assumption A2) appears. We then say that system (5) with the constant removal rate $D^{r}$ is not resilient with respect to the set $\mathscr{K}\left(x_{1}^{r}\right)$ because the trajectory escapes from $\mathscr{K}\left(x_{1}^{r}\right)$ and never comes back (indeed, it converges to the equilibrium point $\left(\lambda_{2}\left(D^{r}\right), 0, s_{i n}-\lambda_{2}\left(D^{r}\right)\right)$.

Let us now study resilience when one allows time-varying removal rate, in terms of viability analysis. Let us recall that the viability kernel of a closed subset $K$ of $\mathbb{R}^{n}$ for a controlled dynamics $\dot{x}=f(x, u(t))$ (with $f: \mathbb{R}^{n} \times \mathbb{R}^{m} \rightarrow \mathbb{R}^{n}$ and $\left.u(t) \in U \subset \mathbb{R}^{m}\right)$, is the largest closed subset $V$ of $K$ such that for any $x_{0} \in V$ there exists $u(\cdot)$ for which the solution with $x(0)=x_{0}$ verifies $x(t) \in K$ for any $t \geq 0$ (see [1]).

Lemma 1: The viability kernel $\operatorname{Viab}\left(\mathrm{cl} \mathscr{K}\left(x_{1}^{r}\right)\right)$ of the closure of the set $\mathscr{K}\left(x_{1}^{r}\right)$ for (5) with controls $D(\cdot) \in\left[D_{m}, D_{M}\right]$ (where $D_{m}, D_{M}$ fulfill (4) and (6)) is given by

$$
\operatorname{Viab}\left(\operatorname{cl} \mathscr{K}\left(x_{1}^{r}\right)\right)=\left[x_{1}^{r}, s_{i n}\right] \times\{0\} .
$$

Proof: From (6), $x_{1}^{r}$ is such that $x_{1}^{r} \in\left(\bar{x}_{1}, s_{i n}-\lambda_{1}\left(D_{m}\right)\right)$. Moreover, Assumptions A1, A2 and condition (4) also imply the inequality $0<\lambda_{2}\left(D_{m}\right)<\lambda_{1}\left(D_{m}\right)<\bar{s}$. Take an initial condition $\left(x_{1,0}, x_{2,0}\right)$ in $\mathrm{cl} \mathscr{K}\left(x_{1}^{r}\right)$.

If $x_{2,0}=0$, the solution of (5) verifies $x_{2}(t)=0$ for any $t>$ 0 and any control $D(\cdot)$. From (6), we can choose a constant control $D$ such that $D_{m} \leq D<\mu_{1}\left(s_{i n}-x_{1}^{r}\right)$, and we observe that (5) with $D(t)=D$ satisfies

$$
\begin{gathered}
x_{1}=x_{1}^{r} \Rightarrow \dot{x}_{1}=\left(\mu_{1}\left(s_{i n}-x_{1}^{r}\right)-D\right) x_{1}^{r}>0, \\
x_{1}=s_{\text {in }} \Rightarrow \dot{x}_{1}=-D s_{\text {in }}<0 .
\end{gathered}
$$

Thus, the constant control $D$ allow the trajectory to stay in the desirable set $\mathscr{K}\left(x_{1}^{r}\right)$.

Assume now that $x_{2,0}>0$. Then, a solution $x(\cdot)$ of (5) associated with an admissible control $D(\cdot)$ verifies $x_{2}(t)>0$ for any time $t \geq 0$. Suppose by contradiction that $x(\cdot)$ stays in $\mathscr{K}\left(x_{1}^{r}\right)$ for any time $t \geq 0$. Then one has $s(t)<s_{i n}-x_{1}^{r}<\bar{s}$ for any time $t \geq 0$. By Assumption A2, one has for any $t \geq 0$, $\mu_{1}(s(t))-\mu_{2}(s(t))<0$ and thus, we deduce the inequality

$$
\begin{aligned}
\dot{s}(t) & >-\mu_{2}(s(t)) x_{1}(t)-\mu_{2}(s(t)) x_{2}(t)+D_{m}\left(s_{i n}-s(t)\right) \\
& =\left(D_{m}-\mu_{2}(s(t))\right)\left(s_{i n}-s(t)\right),
\end{aligned}
$$

for any time $t \geq 0$. Notice that any positive solution $\zeta(\cdot)$ of $\dot{\zeta}=\left(D_{m}-\mu_{2}(\zeta(t))\right)\left(s_{i n}-\zeta(t)\right)$ converges to $\lambda_{2}\left(D_{m}\right)$ when $t \rightarrow+\infty$. From (6) one has $\lambda_{2}\left(D_{m}\right)<s_{i n}-x_{1}^{r}$, hence there exist $t_{1} \geq 0$ and $\underline{s} \in\left(0, \lambda_{2}\left(D_{m}\right)\right)$ such that one has $\zeta(t)>\underline{s}$ for any $t \geq t_{1}$. From the preceding inequality, we deduce that 
$s(\cdot)$ satisfies $s(t) \geq \zeta(t)$ for any time $t \geq 0$. We thus deduce that for any time $t \geq t_{1}$, one has $\mu_{1}(s(t))-\mu_{2}(s(t)) \leq c$ with

$$
c:=\min \left\{\mu_{1}(\sigma)-\mu_{2}(\sigma) ; \sigma \in\left[\underline{s}, s_{\text {in }}-x_{1}^{r}\right]\right\}<0 .
$$

If we differentiate the function $q_{1}:=x_{1} / x_{2}$ w.r.t. $t$, we find that

$$
\dot{q}_{1}=\left(\mu_{1}(s(t))-\mu_{2}(s(t))\right) q_{1},
$$

with $s(t)=s_{i n}-x_{1}(t)-x_{2}(t)$. One then obtains $\dot{q}_{1}<c q_{1}$. Therefore $q_{1}$ decreases to zero and $x_{1}$ as well, leading to a contradiction. We conclude that the only solutions of (5) that stay in $c l \mathscr{K}\left(x_{1}^{r}\right)$ for any time are the ones starting with $x_{2,0}=0$ as was to be proved.

This lemma shows that no control strategy allows the resident species to stay in the desirable set $\mathscr{K}\left(x_{1}^{r}\right)$ in presence of the invader. The dynamics is thus not resilient for the domain $\mathscr{K}\left(x_{1}^{r}\right)$. We are now in a position to introduce a weaker concept of resilience.

Definition 2: Let $x_{1}^{r} \in\left(\bar{x}_{1}, s_{i n}-\lambda_{1}\left(D_{m}\right)\right)$. The system (5) is said to be weakly resilient for the set $\mathscr{K}\left(x_{1}^{r}\right)$ if for any initial condition in $\mathscr{K}\left(x_{1}^{r}\right)$, there exists a control function $D(\cdot)$ with values in $\left[D_{m}, D_{M}\right]$ such that the corresponding solution of (5) satisfies

$$
\text { meas }\left\{t \geq 0 ; x(t) \in \mathscr{K}\left(x_{1}^{r}\right)\right\}=+\infty .
$$

Such a function $D(\cdot)$ will be called a weakly resilient control.

The purpose of the next section is to construct explicitly such control functions.

\section{THE HYBRID CONTROL}

In this section, we shall consider bang-bang controls with values $D_{m}$ or $D_{M}$ (parameters $D_{m}$ and $D_{M}$ satisfying conditions (4) and (6)).

\section{A. Construction of a weakly resilient control}

Let us first give properties of the dynamical system (5) when $D$ is constant $\left(D=D_{m}\right.$ or $\left.D=D_{M}\right)$.

1. For $D=D_{M}$, the "washout" equilibrium $E_{0}:=(0,0)$ is the single equilibrium in $\mathscr{S}$, which is globally asymptotically stable (see Fig. 1).

2. For $D=D_{m}$ : there are three equilibria in $\mathscr{S}: E_{0}, E_{1}:=$ $\left(x_{1}^{m}, 0\right)$ and $E_{2}:=\left(0, x_{2}^{m}\right)$ with $x_{i}^{m}:=s_{i n}-\lambda_{i}\left(D_{m}\right)$. One can easily check that $E_{2}$ is a stable node while $E_{1}$ is a saddle point. By the Theorem of the stable and unstable manifolds, we deduce that the unstable manifold $W^{u}$ of $E_{1}$ connects $E_{1}$ to $E_{2}$, and that the stable manifold of $E_{1}$ belongs to the $x_{2}$ axis (see Fig. 2).

One can also easily check that solutions of (5) with $D=$ $D_{M}$ reach the origin tangentially to the $x_{2}$-axis. Therefore, if $D(t)=D_{M}$ for a sufficiently long time, the state will get so close to the $x_{2}$-axis that a switching to $D=D_{m}$ will produce a further trajectory that will remain close from the stable manifold of $E_{1}$ for a long time and that can then reach the set $\mathscr{K}\left(x_{1}^{r}\right)$, before moving towards the equilibrium $E_{2}$. Then,

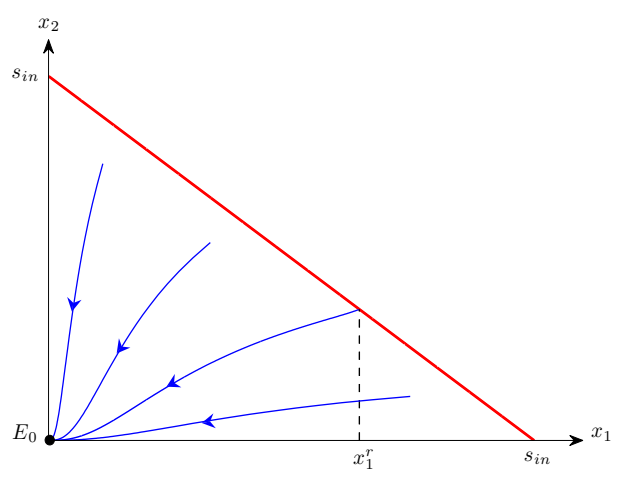

Fig. 1: Phase portrait of (5) with the constant control $D=$ $D_{M}$.

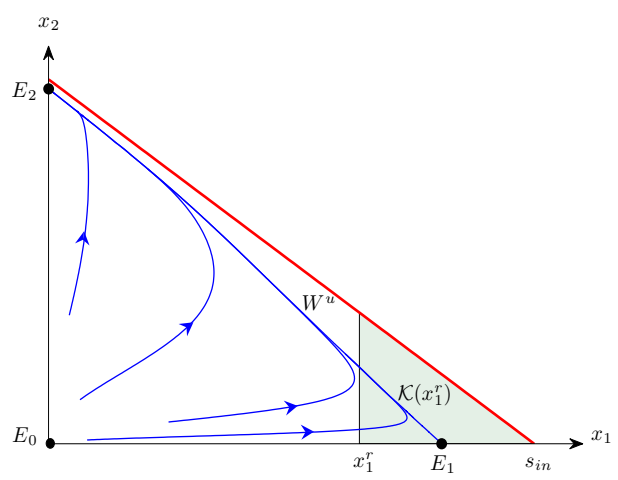

Fig. 2: Phase portrait of 5] with the constant control $D=D_{m}$.

another switch to $D=D_{M}$ when the state leaves $\mathscr{K}\left(x_{1}^{r}\right)$ allows to come back again close to the origin, and so on.

The construction of a weakly resilient control precisely relies on asymptotic properties of (5) with a constant control $D=D_{m}$ or $D=D_{M}$. The next theorem is our main result and formalizes this methodology.

Theorem 2: Assume that $D_{m}$ and $D_{M}$ satisfy (4) and (6). For any $\varepsilon>0$, define the set

$$
\mathscr{E}_{\varepsilon}:=\left(0, \bar{x}_{1}\right] \times(0, \varepsilon]
$$

For $\varepsilon$ small enough and any initial condition $x(0) \in \mathscr{K}\left(x_{1}^{r}\right)$, there exists a piecewise constant function $D(\cdot)$ which alternates the values $D_{M}, D_{m}$ on time intervals $\left[T_{i}, T_{i+1}\right), i \in \mathbb{N}$ satisfying:

$$
T_{0}=0<T_{1}<\cdots<T_{i}<T_{i+1}<\cdots \quad \text { and } \quad \lim _{i \rightarrow \infty} T_{i}=+\infty,
$$

where $x(\cdot)$ is the unique solution of (5) associated with $D(\cdot)$ such that

(i) if $x\left(T_{i}\right) \notin \mathscr{E}_{\varepsilon}$, one has $D(t)=D_{M}$ for $t \in\left[T_{i}, T_{i+1}\right)$ with $T_{i+1}$ defined as the first next entry time into $\mathscr{E}_{\varepsilon}$.

(ii) if $x\left(T_{i}\right) \in \mathscr{E}_{\varepsilon}$, one has $D(t)=D_{m}$ for $t \in\left[T_{i}, T_{i+1}\right)$ and the trajectory $x(\cdot)$ enters into the set $\mathscr{K}\left(x_{1}^{r}\right)$ in finite time ; $T_{i+1}$ is defined as the first next exit time from $\mathscr{K}\left(x_{1}^{r}\right)$.

Moreover, $D(\cdot)$ is a weakly resilient control. 
Du to lack of space, we do not provide the proof of this result (which is long and technical). It mainly consists in studying deep properties (such as image, continuity) of the operator:

$$
\begin{aligned}
\mathscr{O}:\left(0, s_{\text {in }}-x_{1}^{r}\right] & \rightarrow & \left(0, s_{\text {in }}-x_{1}^{r}\right] \\
x_{2,0} & \mapsto & x_{2}\left(T_{2}\right)
\end{aligned}
$$

where $x(\cdot)=\left(x_{1}(\cdot), x_{2}(\cdot)\right)$ denotes the unique solution of (5) for the initial condition $\left(x_{1}^{r}, x_{2,0}\right)$ and the time-varying control $D(\cdot)$ given by Th. 2, parameters $D_{m}, D_{M}, \varepsilon$ being fixed. More details can be found in [10].

Let us underline the fact that the control law given by Th. 2 is an open-loop control, and that the computation of the switching times $T_{i}$ requires the perfect knowledge of the functions $\mu_{j}(\cdot)$. In practice, an open-loop control is not robust with respect to the knowledge of the initial condition and the switching times. Moreover the characteristics of the invasive species is usually not known in advance. However, this control strategy cannot be written as a pure state feedback because when $x_{1}$ reaches the value $x_{1}^{r}$ with $x_{2}>\varepsilon$, one cannot decide if $D=D_{m}$ or $D=D_{M}$ without knowing the past, i.e., if $x_{1}$ is increasing or decreasing.

\section{B. Synthesis with a hybrid control}

Instead, we propose a hybrid controller associated with a logic-based switching algorithm represented by a piecewise signal $\sigma(\cdot)$ that can take three possible states denoted by $\chi_{1}, \chi_{2}$ and $\chi_{3}$. The transitions between these three states is illustrated on Fig. 3

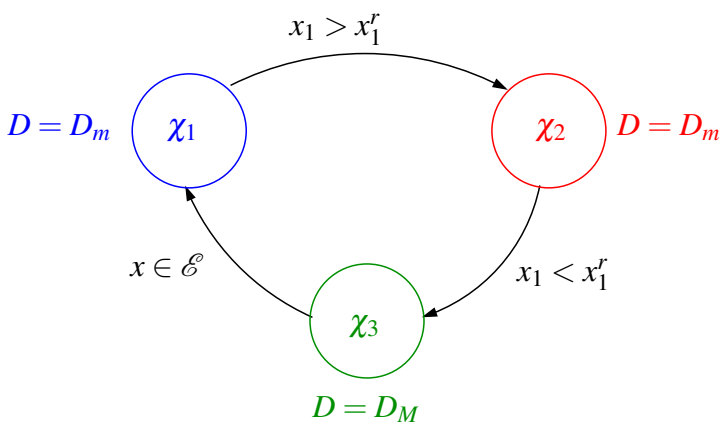

Fig. 3: The logic-based algorithm associated with the hybrid control.

One has the following result.

Proposition 3: Let $\sigma(\cdot): \mathbb{R}_{+} \rightarrow\left\{\chi_{1}, \chi_{2}, \chi_{3}\right\}$ be such that

$$
\begin{aligned}
\sigma(t) & =g\left(x_{1}(t), \sigma\left(t_{-}\right)\right) \\
= & \begin{cases}\chi_{2} & \text { if } \sigma\left(t_{-}\right)=\chi_{1} \text { and } x_{1}(t)>x_{1}^{r}, \\
\chi_{3} & \text { if } \sigma(t-)=\chi_{2} \text { and } x_{1}(t)<x_{1}^{r}, \\
\chi_{1} & \text { if } \sigma\left(t_{-}\right)=\chi_{3} \text { and } x(t) \in \mathscr{E}_{\varepsilon}, \\
\sigma\left(t_{-}\right) & \text {otherwise, }\end{cases}
\end{aligned}
$$

with $\sigma(0)=\chi_{3}$. Then, the $\sigma$-feedback:

$$
D(\sigma(t))= \begin{cases}D_{m} & \text { if } \sigma(t) \in\left\{\chi_{1}, \chi_{2}\right\}, \\ D_{M} & \text { if } \sigma(t)=\chi_{3} .\end{cases}
$$

guarantees the weak resilience of the dynamics with the control (8).
Proof: This automata is a simple way to memorize if the state $x_{1}$ is entering or leaving the set $\mathscr{K}\left(x_{1}^{r}\right)$. One can easily see that solutions of the coupled dynamics

$$
\begin{aligned}
& \dot{x}(t)=f(x(t), D(\sigma(t))), \quad x(0) \in \mathscr{K}\left(x_{1}^{r}\right), \\
& \sigma(t)=g\left(x_{1}(t), \sigma\left(t_{-}\right)\right), \quad \sigma(0)=\chi_{3},
\end{aligned}
$$

(see for instance [17] for the solution concept) are such that the times $T_{i}$ given by Th. 2 correspond exactly to the ones of $\sigma$ (when switching from $\chi_{2}$ to $\chi_{3}$ or from $\chi_{3}$ to $\chi_{1}$ ). Provided that $\varepsilon$ is small enough and that $D_{m}$ and $D_{M}$ satisfy (4) and (6), Th. 2 then guarantees weak resilience of the hybrid controller (8).

In [10], it has been shown that there exist periodic solutions of (5) associated with the control given in Th. 2 . It has been conjectured that for $\varepsilon$ sufficiently small, the operator $\mathscr{O}: x_{2,0} \mapsto x_{2}\left(T_{2}\right)$, where $T_{2}$ is given by Th. 2 for the initial condition $\left(x_{1}^{r}, x_{2,0}\right)$, is contractive. Then, under this condition, it has been proved that any trajectory with initial condition in $\mathscr{K}\left(x_{1}^{r}\right)$ and the weakly resilient control given by Th. 2 converges asymptotically to a unique periodic solution $x^{\dagger}(\cdot)$ with a period $T^{\dagger}$, up to a time shift.

Remark 1: The synthesis of the hybrid controller requires the single choice of the parameters $D_{m}$ and $D_{M}$ that satisfy (4) and (6) (which are quite loose conditions) and $\varepsilon>0$ small enough. Therefore, it does not require the precise knowledge of the functions $\mu_{i}$ to ensure weak resilience against a species that fulfills Assumption A2, in contrast with more sophisticated approaches, such as model predictive control, which rely on more knowledge on the growth functions. Let us also underline that the controller switches between an environment that is unfavorable to both species $\left(D=D_{M}\right)$ and an environment which is favorable to the invasive species $\left(D=D_{m}\right)$. It is then not intuitive that the resident species could be dominant most of the time under such a switching. This property is strongly linked to the choice of $\varepsilon$ that has simply to be sufficiently small, although we are not able to provide an explicit bound.

\section{NUMERICAL SIMULATIONS}

Numerical simulations have been performed to illustrate the behavior of the trajectories generated by the hybrid controller and the role of its parameters on the proportion of time spent in the desired set $\mathscr{K}\left(x_{1}^{r}\right)$, as well as their impact on the productivity of the resident species.

We consider two growth functions of Monod's type:

$$
\mu_{1}(s):=\frac{0.5 s}{5+s} ; \mu_{2}(s):=\frac{0.16 s}{0.13+s},
$$

with $s_{\text {in }}=5$, see Fig. 4 .

Let us first notice that in absence of species 2, the hybrid controller gives $D(t)=D_{m}$. We have simulated an invasion of species 2 at time $t_{I}=50$ with a sudden input of a small amount of concentration $x_{2}\left(t_{I}^{+}\right)=0.2$. Before the invasion, one can observe that the system is at quasi-steady state and after the invasion, the control $D(t)$ alternates between $D_{m}$ and $D_{M}$. The solution finally converges asymptotically to a periodic solution $x^{\dagger}(\cdot)$. 


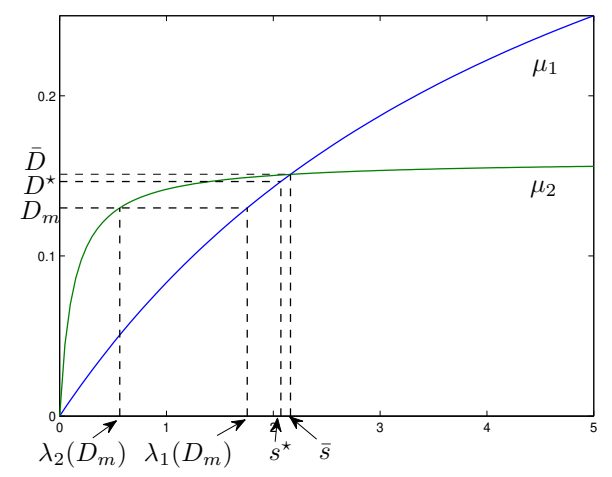

Fig. 4: Graphs of the functions $\mu_{1}$ and $\mu_{2}$ with $s_{i n}=5$.

\section{A. Proportion of time spent in $\mathscr{K}\left(x_{1}^{r}\right)$}

On Figs. 5 and 6, two different values of $\varepsilon$ have been chosen. In Table I] we also present for different values of $\varepsilon$,

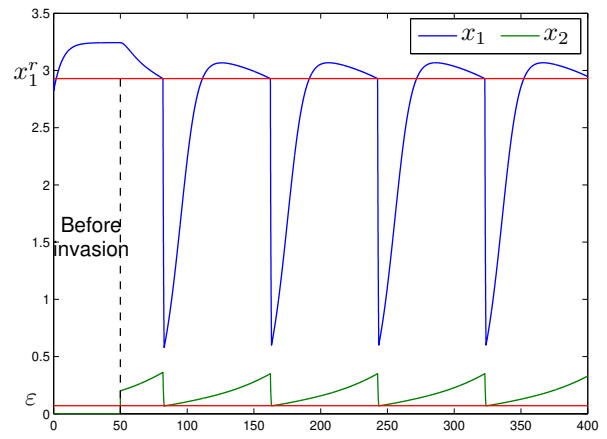

Fig. 5: Time courses for $\varepsilon=0.1, D_{m}=0.13$ and $D_{M}=2$.

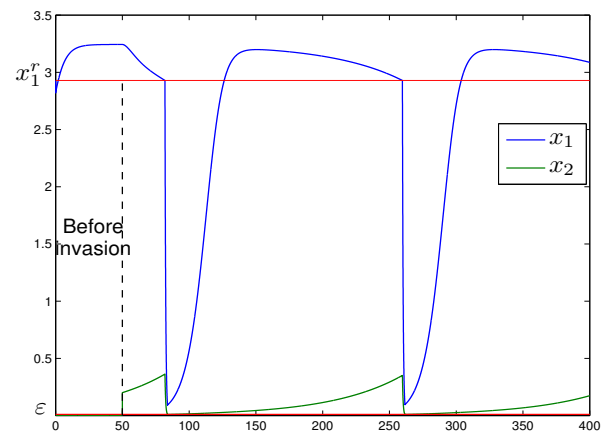

Fig. 6: Time courses for $\varepsilon=0.01, D_{m}=0.13$ and $D_{M}=2$.

the proportion of time spent by the periodic solution $x^{\dagger}(\cdot)$ in the desirable set $\mathscr{K}\left(x_{1}^{r}\right)$, during one period (other parameters being fixed). When $\varepsilon$ decreases, one observes that the period increases, as it requires a longer time for the trajectory to reach the set $\mathscr{E}_{\varepsilon}$. Interestingly, one can also observe that the proportion of time spent in $\mathscr{K}\left(x_{1}^{r}\right)$ increases when $\varepsilon$ decreases. Indeed, when $\varepsilon$ is small, the trajectory gets close to the stable manifold of the saddle equilibrium $E_{1}$ when
$D=D_{m}$ (which is the $x_{1}$-axis) and therefore the state stays a long time in the vicinity of $E_{1}$ which belongs to $\mathscr{K}\left(x_{1}^{r}\right)$.

\begin{tabular}{|c|c|c|c|c|}
\hline$\varepsilon$ & period & $\begin{array}{c}\text { \% of time } \\
\text { in } \mathscr{K}\left(x_{1}^{r}\right)\end{array}$ & $\begin{array}{c}\text { average } \\
\text { productivity }\end{array}$ & $\begin{array}{c}\text { \% of } \\
\text { loss }\end{array}$ \\
\hline 0.001 & 293 & 78.50 & 0.3562 & 15.51 \\
0.01 & 177 & 75.20 & 0.3596 & 14.71 \\
0.07 & 80.3 & 63.65 & 0.3674 & 12.86 \\
0.15 & 40.5 & 36.67 & 0.3755 & 10.93 \\
\hline
\end{tabular}

TABLE I: Asymptotic performances with $D_{m}=0.13$ and $D_{M}=2$.

\section{B. Productivity of the resident species}

Let us consider the productivity $P$ of the species 1 alone, as the quantity produced per unit of volume and time when the system is at steady steady state. It is given by

$$
P:=D x_{1}^{e q}(D),
$$

where $x_{1}^{e q}(D)$ is the steady state associated with a constant control $D$ (or equivalently $P=\mu_{1}\left(s^{e q}\right)\left(s_{i n}-s^{e q}\right)$ ). The largest value of $P$ is then obtained when the substrate concentration at steady state that maximizes the function $s \mapsto \mu_{1}(s)\left(s_{\text {in }}-s\right)$ at a certain $s^{\star} \in\left(0, s_{i n}\right)$. Then, the maximal productivity is obtained for the dilution rate $D^{\star}=\mu_{1}\left(s^{\star}\right)$ and one has $x_{1}^{\star}=$ $s_{\text {in }}-s^{\star}$. For the chosen value of $s_{i n}$, the maximal productivity of species 1 is obtained for $P_{\max } \simeq 0.4289$ (see Fig. 7). We

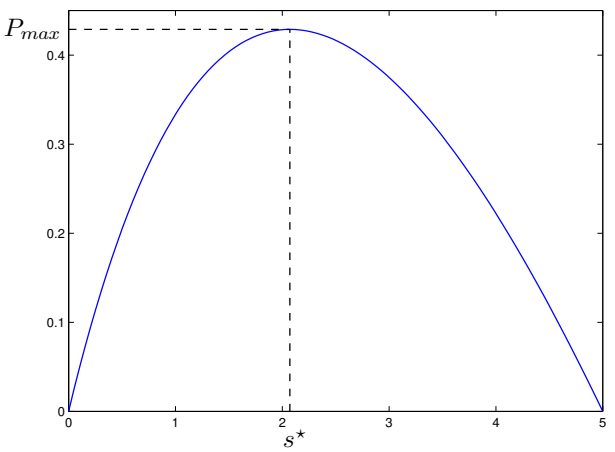

Fig. 7: Graph of the function $s \mapsto \mu_{1}(s)\left(s_{i n}-s\right)$ with $s_{i n}=5$.

have then chosen a threshold $x_{1}^{r}$ equal to $x_{1}^{\star}$ and a value of $D_{m}$ close to $D^{\star}$ (such that inequality (6) fulfills), so that it does not impact too much the productivity of species 1 as long as species 2 is absent (see Fig. 4). In absence of species 2, as the controller takes the value $D_{m}$, the productivity of species 1 alone at steady state is $P_{m}:=D_{m} x_{1}^{m} \simeq 0.4216$ (which is quite close to the maximal one).

In presence of species 2 , we have considered the average productivity $\bar{P}:=\frac{1}{T^{\dagger}} \int_{0}^{T^{\dagger}} D(t) x_{1}^{\dagger}(t) d t$ of species 1 over a period of the asymptotic periodic solution $x^{\dagger}(\cdot)$, to be compared with the productivity of species 1 alone (see Table II). The percentage of loss of productivity $\frac{P_{m}-\bar{P}}{P_{m}}$ is also indicated. It is clear that the presence of the species 2 impacts the productivity of species 1 , as there is a new consumer of the resource. However, one can see that it is possible to maintain a relatively low decrease of productivity, under the condition that the parameter $\varepsilon$ is not too small. 
In conclusion, as already known in the literature in the context of single species (see for instance [6]), one can appreciate the trade-off between having a high productivity and maintaining a high conversion. The parameter $\varepsilon$ is thus a lever of choice for the practitioners.

\section{CONCLUSiON}

In this work we have proposed a hybrid controller that allows weak resilience in the chemostat model. This controller switches between bounds $D_{m}$ and $D_{M}$, so that the corresponding solution enters infinitely many times into a desirable subset (while each bound is unfavorable to resilience when considered alone). One of the main advantages of this controller is that it does not require the precise knowledge of the growth characteristics of the invader and can cope with a large variety of unknown invading species. In practice, one can simply apply this controller even in absence of invading species, guaranteeing a robustness of the performances against possible future invasions. We have also shown its features in terms of time spent in the desirable subset and productivity thanks to an adequate choice of the bounds $D_{m}$ and $D_{M}$ and of the parameter $\varepsilon$, although we do not know if the bang-bang strategy is optimal for the so-called "time of crisis" (see [7], [8], [9]):

$$
\inf _{D(\cdot)} \text { meas }\left\{t \in[0, T] ; x(t) \notin \mathscr{K}\left(x_{1}^{r}\right)\right\} \text {. }
$$

This amounts to minimize w.r.t. admissible controls the time spent by trajectories outside the desirable set $\mathscr{K}\left(x_{1}^{r}\right)$ either over a finite given horizon $[0, T]$ (which is more relevant in the present context), or over $[0,+\infty)$ using the notion of finitely optimal control as in [4]. This could be the matter of a future work. When practitioners possess more information on the growth functions (such as average and variability, especially on the possible invasive species), it would be interesting to compare the performances of the proposed controller with more classical approaches such as model predictive control. This could be also studied in a future work. Finally, an extension of this work could deal with different removal rates of species.

\section{REFERENCES}

[1] J.P. AuBIn, Viability Theory, Birkäuser, Boston, 1991.

[2] R. ARIS AND A.E. HuMPHREY, Dynamics of a chemostat in which two organisms compete for a common substrate, Biotechnology and Bioengineering, 19, 1375-1386, 1977.

[3] R. Armstrong and R. McGehee, Competitive exclusion, American Naturalist, 115, 151-170, 1980.

[4] S. AseEv, V. Veliov, Maximum principle for infinite-horizon optimal control problems under weak regularity assumptions, Tr. Inst. Mat. Mekh. 20 (2014), no. 3, pp. 41-57.

[5] G. BAStin AND D. Dochain, On-line estimation and adaptive control of bioreactors, Elsevier Sc. Publishers, 1990.

[6] G. Bastin, D. Nesic, Y. Tan and I. Mareels, On extremum seeking in bioprocesses with multivalued cost functions, Biotechnol Progress, 25, 683-689, 2009.

[7] T. BAYEN AND A. RAPAPORT, About Moreau-Yosida regularization of the minimal time crisis problem, J. Convex Anal. 23(1), 263-290, 2016.

[8] T. BAYEN AND A. RAPAPORT, About the minimal time crisis problem, ESAIM: Proceedings and Surveys, 57, 1-11, 2017.
[9] T. BAYEN AND A. RAPAPORT, Minimal time crisis versus minimum time to reach a viability kernel: a case study in the prey-predator model, Optimal Control Applications and Methods, 40(2), 330-350, 2019.

[10] T. BAYEN, A. RAPAPORT AND F.Z-. TANI, Weak resilience of the chemostat model to a species invasion with non-autonomous removal rates, pre-publication https://hal.archives-ouvertes.fr/hal-02068276 2019.

[11] G.J. Butler, S.B. HSU AND P.E. Waltman, A mathematical model of the chemostat with periodic washout rate, SIAM J. Appl. Math., 45, 435-449, 1985.

[12] G.J. BUTLER AND G.S.K. Wolkowicz, A mathematical model of the chemostat with a general class of functions describing nutrient uptake, SIAM Journal on Applied Mathematics, 45 (1), 138-151, 1985.

[13] J.P. GRIVET, Nonlinear population dynamics in the chemostat, IEEE Computing in Science \& Engineering, 3, 48-55, 2001.

[14] J.P. Grover, Resource Competition, Population and Community Biology Series, 19, Chapman \& Hall, New York, 1997.

[15] J. HARMAnd, C. LOBRY, A. RAPAPORT AND T. SARI, The chemostat, mathematical theory of continuous culture of micro-organisms, ISTE Press, London, 2017.

[16] S.B. HSU, S.HubBELl AND P. WALtMAn, A mathematical theory for single-nutrient competition in continuous cultures of micro-organisms, SIAM Journal on Applied Mathematics, 32, 366-383, 1977.

[17] D. Liberzon, Switching in Systems and Control, Birkäuser, Boston, 2003.

[18] N. Panikov, Microbial Growth Kinetics, Chapman \& Hall, New York, 1995.

[19] H. L. Sмith, Competitive coexistence in an oscillating chemostat, SIAM Journal on Applied Mathematics, 40, 498-522, 1981.

[20] H. SMith AND P. WALTMAn, The theory of chemostat, dynamics of microbial competition, Cambridge Studies in Mathematical Biology, Cambridge University Press, 1995.

[21] G. STEPhanopoulos, R. ARIS AND A. Frederickson, A stochastic analysis of the growth of competing microbial populations in a continuous biochemical reactor, Mathematical Biosciences, 45, 99$135,1979$.

[22] G. Wolkowicz AND X. ZhaO, $N$-species competition in a periodic chemostat, Differential Integral Equations 11, 465-491, 1998. 